\title{
THE LAW OF THE ITERATED LOGARITHM IN UNIFORMLY CONVEX BANACH SPACES
}

\author{
BY \\ MICHEL LEDOUX
}

\begin{abstract}
We give necessary and sufficient conditions for a random variable $X$ with values in a uniformly convex Banach space $B$ to satisfy the law of the iterated logarithm. Precisely, we show that a $B$-valued random variable $X$ satisfies the (compact) law of the iterated logarithm if and only if $E\left\{\|X\|^{2} / L_{2}\|X\|\right\}<\infty$, the family $\left\{\left|x^{*}(X)\right|^{2} ; x^{*} \in B^{*},\left\|x^{*}\right\|=1\right\}$ is uniformly integrable and $S_{n} / a_{n} \rightarrow 0$ in probability.
\end{abstract}

1. Introduction. Let $B$ be a Banach space with topological dual $B^{*}$ and norm $\|\cdot\|$. By random variable with values in $B$, we mean a measurable map $X$ from some probability space $(\Omega, \mathcal{F}, P)$ into $B$ such that the image of $P$ by $X$ defines a Radon probability measure on the Borel $\sigma$-field of $B$. We may assume equivalently that $X$ takes almost surely its values in a separable subspace of $B$. Given a $B$-valued random variable $X$, we denote by $\left(X_{n}\right)_{n \in \mathbf{N}}$ a sequence of independent copies of $X$ and let, as usual, $S_{n}=X_{1}+\cdots+X_{n}$. We write $L t$ to denote the function $\max (1, \log t)$ and use $L_{2} t$ to denote $L(L t)\left(t \in \mathbf{R}_{+}\right)$. We set further $a_{n}=\left(2 n L_{2} n\right)^{1 / 2}$. The random variable $X$ is said to satisfy the bounded law of the iterated logarithm (LIL) if the sequence $\left(S_{n} / a_{n}\right)_{n \in \mathbf{N}}$ is bounded with probability one, and the compact LIL if this sequence is almost surely relatively compact in $B$. It is well known that if $X$ satisfies the compact LIL, there is a compact convex and symmetric set $K$ in $B$ such that

$$
\lim _{n \rightarrow \infty} d\left(\frac{S_{n}}{a_{n}}, K\right)=0 \quad \text { and } C\left(\frac{S_{n}}{a_{n}}\right)=K \quad \text { w.p.1, }
$$

where $d(x, K)=\inf \{\|x-y\| ; y \in K\}$ and $C\left(S_{n} / a_{n}\right)$ denotes all limit points of the sequence $\left(S_{n} / a_{n}\right)_{n \in \mathbf{N}}[\mathbf{1 6}]$; the set $K$ is often described as the unit ball of the reproducing kernel Hilbert space determined by the covariance structure of $X$ (see $[\mathbf{1 6}, \mathbf{6}]$ for more details on this aspect).

Let us now recall and briefly describe the usual necessary conditions required of $X$ to satisfy the bounded LIL (for simplicity, we restrict ourselves to a description of the bounded LIL in this introduction; see $\S 3$ for the corresponding results concerning the compact LIL). If the sequence $\left(S_{n} / a_{n}\right)_{n \in \mathbf{N}}$ is bounded with probability one, the same is true for $\left(X_{n} / a_{n}\right)_{n \in \mathbf{N}}$, which consists of independent variables, and hence, by the Borel-Cantelli lemma, we have $\sum_{n} P\left\{\|X\|>M a_{n}\right\}<\infty$ for some $M>0$ and thus

$$
E\left\{\|X\|^{2} / L_{2}\|X\|\right\}<\infty .
$$

Received by the editors April 24, 1985.

1980 Mathematics Subject Classification. Primary 60B12, 60B11; Secondary 46B20.

Key words and phrases. Law of the iterated logarithm, uniformly convex Banach spaces, smooth norm. 
As a second necessary condition, the scalar LIL implies that

$$
\text { for each } x^{*} \text { in } B^{*}, \quad E\left\{\left|x^{*}(X)\right|^{2}\right\}<\infty,
$$

or, equivalently,

$$
\sigma=\sigma(X)=\sup _{\left\|x^{*}\right\|=1}\left(E\left\{\left|x^{*}(X)\right|^{2}\right\}\right)^{1 / 2}<\infty,
$$

which follows easily from a closed graph argument, and $E\{X\}=0$ which is well defined under (1.1). Finally, if $X$ satisfies the bounded LIL, it is necessary that

the sequence $\left(S_{n} / a_{n}\right)_{n \in \mathbf{N}}$ be stochastically bounded.

(Note that (1.3) includes the (trivial) mean zero property of $X$.) It is not known whether or not these three necessary conditions are also sufficient for $X$ to satisfy the bounded LIL in general; if it were the case, it would reduce the almost sure statement of the LIL to a stochastic statement which, under necessary moment conditions, is close to the central limit theorem (CLT) statement. A collection of results supports the hope that they are. J. Kuelbs $[\mathbf{1 7}]$ showed that when $E\left\{\|X\|^{2}\right\}<\infty$ (which implies (1.1) and $\sigma(X)<\infty) X$ satisfies the bounded LIL iff (1.3) holds; on the other hand, the relation between the CLT and the LIL $[\mathbf{2 5 , 6 , 7 ]}$ shows that when $\left(S_{n} / \sqrt{n}\right)_{n \in \mathbf{N}}$ is stochastically bounded (a stronger hypothesis than (1.2) and (1.3)), $X$ satisfies the bounded LIL iff (1.1) holds. However, neither $E\left\{\|X\|^{2}\right\}<\infty$ nor the CLT are necessary for $X$ to verify the LIL in general. Besides these general results, conditions (1.1), (1.2) and (1.3) are known to be sufficient for $X$ to satisfy the bounded LIL in certain type 2 spaces. Recall that a Banach space $B$ is of type $p(1<p \leq 2)[\mathbf{2 2}]$ if there exists a positive constant $C$ such that

$$
E\left\{\left\|\sum_{i} \varepsilon_{i} x_{i}\right\|\right\} \leq C\left(\sum_{i}\left\|x_{i}\right\|^{p}\right)^{1 / p} \quad \text { for all }\left(x_{i}\right) \subseteq B,
$$

where $\left(\varepsilon_{i}\right)$ denotes a Rademacher sequence, i.e. a sequence of independent random variables taking the values \pm 1 with probability $\frac{1}{2}$. Note that in type 2 spaces, (1.3) (actually $S_{n} / a_{n} \rightarrow 0$ in probability) follows immediately from the integrability condition (1.1), and $E\{X\}=0$ so that in these spaces the results on the LIL do not involve condition (1.3). It was first proved by V. Goodman, J. Kuelbs and J. Zinn [6] that (1.1) and (1.2) are necessary and sufficient for a mean zero Hilbert space valued random variable $X$ to satisfy the bounded LIL. This characterization was then extended to more general type 2 spaces (basically the $L_{p}$ spaces with $2 \leq p<\infty)$, in [1] , in spaces satisfying some upper Gaussian comparison principle, in [18], in 2-uniformly smoothable Banach spaces. The proof of these results rests on some differentiability properties of smooth norms allowing an efficient use of the weak moment condition (1.2), which is the main difficulty in proving LIL results. The use of smooth norms in the context of the LIL goes back to [15].

In this paper, we use conditions (1.1), (1.2) and (1.3) to characterize the bounded LIL in uniformly convex Banach spaces; as a corollary, we also obtain the compact LIL as stated in the abstract. A Banach space $B$ is uniformly convex provided that for every $\varepsilon>0$ there is a $\delta(\varepsilon)>0$ such that if $\|x\|=\|y\|=1$ and $\|x-y\| \geq$ $\varepsilon, 1-\|(x+y) / 2\| \geq \delta(\varepsilon)$. $L_{p}$ spaces for $1<p<\infty$ are uniformly convex. According to a fundamental result of G. Pisier [23], a uniformly convex Banach 
space admits an equivalent norm whose corresponding modulus of convexity $\delta(\varepsilon)$ satisfies $\delta(\varepsilon) \geq L \varepsilon^{q}$ for some $q<\infty$ and positive constant $L$. The dual statement yields another equivalent norm with a modulus of smoothness of power type $p$ for some $p>1$. In the study of the LIL, we will make use of these smoothness (and related differentiation) properties together with a conditioning argument due to $\mathrm{V}$. V. Yurinskii $[\mathbf{2 8}]$ to define a real valued martingale whose differences can be nicely controlled, allowing thus the use of some exponential inequalities for martingales. Lemma 2.1 below is the crucial step in this framework and is described in $\S 2$. $\S 3$ contains the proof of our main result providing necessary and sufficient conditions for the LIL in uniformly convex spaces. In $\S 4$, we show that if $X$ is a Banach space valued random variable satisfying the LIL and $\xi$ a real valued random variable independent of $X$ and belonging to the Lorentz space $L_{2,1}$, then the product $\xi X$ still satisfies the LIL, the integrability condition on $\xi$ being the best possible in general. The corresponding results for the CLT $[\mathbf{5}, \mathbf{2 0}]$ show that this property must hold in case conditions (1.1), (1.2) and (1.3) were necessary and sufficient for $X$ to satisfy the LIL in any Banach space. Our results were announced in [19].

2. Preliminary results; the crucial lemma. A Banach space $B_{1}$ is finitely representable in a Banach space $B_{2}$ if for every $\varepsilon>0$ and every finite dimensional subspace $F_{1}$ of $B_{1}$ there is a subspace $F_{2}$ of $B_{2}$ such that $d\left(F_{1}, F_{2}\right) \leq 1+\varepsilon$, where $d$ denotes the Banach-Mazur distance between Banach spaces. Let $P$ be a property concerning Banach spaces; a Banach space $B$ has the property super$P$ if all the Banach spaces which are finitely representable in $B$ have property $P$. Super properties were introduced in $[\mathbf{1 1}]$ where superreflexivity is studied. Uniform convexity and superreflexivity are intimately connected. Recall that a Banach space $B$ is uniformly convex if the modulus of convexity

$$
\delta(\varepsilon)=\inf \{1-\|(x+y) / 2\| ;\|x\|=\|y\|=1,\|x-y\| \geq \varepsilon\}
$$

is strictly positive for each $\varepsilon$ in $(0,2]$. Uniformly convex spaces are superreflexive. According to a fundamental result of P. Enflo [4], every superreflexive Banach space admits an equivalent uniformly convex norm. Using martingales, G. Pisier $[\mathbf{2 3}]$ considerably strengthened this result and showed the existence in every superreflexive space of an equivalent norm whose corresponding modulus of convexity is of power type; i.e., $\delta(\varepsilon) \geq L \varepsilon^{q}$ for every $\varepsilon$ in $(0,2]$ and some $q<\infty$ and positive constant $L$. These results have dual analogues (via Lindenstrauss' duality formula; cf. [21]) concerning the modulus of smoothness. The modulus of smoothness of a Banach space $B$ is defined as

$$
\rho(t)=\sup \left\{\frac{1}{2}(\|x+t y\|+\|x-t y\|)-1 ;\|x\|=\|y\|=1\right\}, \quad t>0 ;
$$

$B$ is uniformly smooth if $\lim _{t \rightarrow 0} \rho(t) / t=0$. Uniformly smooth spaces are superreflexive, and G. Pisier's result indicates that any superreflexive Banach space $B$ admits an equivalent norm for which the modulus of smoothness $\rho$ satisfies $\rho(t) \leq K t^{p}$ for all $t>0$ and some $p>1$ and positive constant $K$. Such a norm will be called $p$-smooth in the sequel, and $B$ is said to be $p$-uniformly smoothable; $p$-smoothable spaces are of type $p$ (cf. $[\mathbf{2}, \mathbf{2 3}])$.

Given a Banach space $B$ with a $p$-smooth norm $\|\cdot\|$ for some $p>1$, let us denote by $D$ the derivative of this norm uniformly Fréchet-differentiable away from the origin (cf. $\left[\mathbf{3}\right.$ or 14]) and define a map $F_{p}$ from $B$ into $B^{*}$, the dual space of $B$, 
by $F_{p}(x)=\|x\|^{p-1} D(x /\|x\|)$ for $x \neq 0, F_{p}(0)=0$. As an element of $B^{*}, F_{p}(x)$ is of norm $\|x\|^{p-1}$ for all $x$ in $B$. As was shown by J. Hoffmann-Jørgensen [10], the power type estimate of the modulus of smoothness of this $p$-smooth norm can be translated in an equivalent form on the derivative $F_{p}$ by a Hölder condition

$$
\left\|F_{p}(x)-F_{p}(y)\right\| \leq C\|x-y\|^{p-1} \text { for all } x, y \text { in } B
$$

and some constant $C>0$. Since

$$
\|x+y\|^{p}-\|x\|^{p}=p \int_{0}^{1} F_{p}(x+t y)(y) d t,
$$

we deduce from (2.1) that

$$
\left|\|x+y\|^{p}-\|x\|^{p}\right| \leq p\left|F_{p}(x)(y)\right|+C\|y\|^{p} \quad \text { for all } x, y \text { in } B .
$$

This decomposition of $p$-smooth norms will be the first step in the method of proof of our main result; applied to independent random variables, it will allow, together with Fubini's theorem, an efficient use of the weak moment hypotheses (1.2). Formula (2.2), however, needs to be combined with a conditioning property; the lemma below which describes this argument is the crucial point in our approach. $B$ denotes as before a $p$-uniformly smooth Banach space for some $p>1$ with a $p$ smooth norm $\|\cdot\|$ for which $(2.2)$ holds.

Lemma 2.1. Let $\left(Y_{i}\right)_{1 \leq i \leq n}$ be a finite sequence of independent bounded $B$ valued random variables and let $S=\sum_{i=1}^{n} Y_{i}$. For each $i=1, \ldots, n$, denote by $\mathcal{F}_{i}$ the $\sigma$-field generated by the random variables $Y_{1}, \ldots, Y_{i} ; \mathcal{F}_{0}$ is the trivial field. Then $\|S\|^{p}-E\left\{\|S\|^{p}\right\}$ can be written as a martingale

$$
\|S\|^{p}-E\left\{\|S\|^{p}\right\}=\sum_{i=1}^{n} d_{i}
$$

whose differences $d_{i}=E\left\{\| S||^{p} \mid \mathcal{F}_{i}\right\}-E\left\{\left.|| S\right|^{p} \mid \mathcal{F}_{i-1}\right\}$ satisfy, for each $i=1, \ldots, n$,

$$
\left|d_{i}\right| \leq p\left(\left\|Y_{i}\right\|+E\left\{\left\|Y_{i}\right\|\right\}\right) E\left\{\left\|S-Y_{i}\right\|^{p-1} \mid \mathcal{F}_{i-1}\right\}+C\left(\left\|Y_{i}\right\|^{p}+E\left\{\left\|Y_{i}\right\|^{p}\right\}\right)
$$

and

$$
E\left\{d_{i}^{2} \mid \mathcal{F}_{i-1}\right\} \leq 2 p^{2} E\left\{F_{p}^{2}\left(S-Y_{i}\right)\left(Y_{i}\right) \mid \mathcal{F}_{i-1}\right\}+2 C^{2} E\left\{\left\|Y_{i}\right\|^{2 p}\right\} .
$$

Proof. Let $i$ be fixed. V. V. Yurinskii's observation [28], which follows by independence, indicates that

$$
d_{i}=\left(E\left\{\cdot \mid \mathcal{F}_{i}\right\}-E\left\{\cdot \mid \mathcal{F}_{i-1}\right\}\right)\left(\|S\|^{p}-\left\|S-Y_{i}\right\|^{p}\right) .
$$

Therefore by $(2.2)$

$$
\begin{aligned}
\left|d_{i}\right| & \leq\left(E\left\{\cdot \mid \mathcal{F}_{i}\right\}+E\left\{\cdot \mid \mathcal{F}_{i-1}\right\}\right)\left(\left|\|S\|^{p}-\left\|S-Y_{i}\right\|^{p}\right|\right) \\
& \leq\left(E\left\{\cdot \mid \mathcal{F}_{i}\right\}+E\left\{\cdot \mid \mathcal{F}_{i-1}\right\}\right)\left(p\left|F_{p}\left(S-Y_{i}\right)\left(Y_{i}\right)\right|+C\left\|Y_{i}\right\|^{p}\right) \\
& \leq\left(E\left\{\cdot \mid \mathcal{F}_{i}\right\}+E\left\{\cdot \mid \mathcal{F}_{i-1}\right\}\right)\left(p\left\|S-Y_{i}\right\|^{p-1}|| Y_{i}\|+C\| Y_{i} \|^{p}\right)
\end{aligned}
$$

and hence the announced majorization for $\left|d_{i}\right|$ since again

$$
E\left\{|| S-Y_{i} \|^{p-1} \mid \mathcal{F}_{i}\right\}=E\left\{\| S-\left.Y_{i}\right|^{p-1} \mid \mathcal{F}_{i-1}\right\} .
$$


In the same way

$$
\begin{aligned}
E\left\{d_{i}^{2} \mid \mathcal{F}_{i-1}\right\} & \leq E\left\{||\left|S\left\|^{p}-\right\| S-Y_{i} \|^{p}\right|^{2} \mid \mathcal{F}_{i-1}\right\} \\
& \leq E\left\{\left(p\left|F_{p}\left(S-Y_{i}\right)\left(Y_{i}\right)\right|+C|| Y_{i} \|^{p}\right)^{2} \mid \mathcal{F}_{i-1}\right\}
\end{aligned}
$$

and this completes the proof of the lemma.

Provided with this lemma, we shall study the LIL in uniformly convex spaces through the martingales $\left\|S_{n}\right\|^{p}-E\left\{\left\|S_{n}\right\|^{p}\right\}$ for a given equivalent $p$-smooth norm $\|\cdot\|$ for some $p>1$, the centerings $E\left\{\left\|S_{n}\right\|^{p}\right\}$ being estimated under (1.3) (this procedure will actually be applied to partial sums associated to several truncations of $X$ ). Some of these martingales will be studied using classical exponential inequalities like the following one, which we prove for the sake of completeness.

LEMMA 2.2. Let $\sum_{i=1}^{n} d_{i}$ be a sum of martingale differences with respect to the filtration $\left(\mathcal{F}_{i}\right)_{i \leq n}$ such that, for each $i=1, \ldots, n,\left|d_{i}\right| \leq c$ and $E\left\{d_{i}^{2} \mid \mathcal{F}_{i-1}\right\} \leq b^{2}$ for positive constants $c$ and $b$. Then, for every $t$ and $\lambda>0$,

$$
P\left\{\sup _{i \leq n} \sum_{j=1}^{i} d_{j}>t\right\} \leq \exp \left(-\lambda t+\frac{\lambda^{2}}{2} \cdot n b^{2} e^{\lambda c}\right) .
$$

ProOF. By the submartingale maximal inequality, for each $t$ and $\lambda>0$,

$$
P\left\{\sup _{i \leq n} \sum_{j=1}^{i} d_{j}>t\right\} \leq \exp (-\lambda t) E\left\{\exp \lambda\left(\sum_{i=1}^{n} d_{i}\right)\right\} .
$$

Now

$$
E\left\{\exp \lambda\left(\sum_{i=1}^{n} d_{i}\right)\right\}=E\left\{\exp \lambda\left(\sum_{i=1}^{n-1} d_{i}\right) E\left\{\exp \lambda d_{n} \mid \mathcal{F}_{n-1}\right\}\right\} .
$$

A series expansion of the exponential function and simple use of the hypotheses easily imply that

$$
\begin{aligned}
E\left\{\exp \lambda d_{n} \mid \mathcal{F}_{n-1}\right\} & =1+\frac{\lambda^{2}}{2 !} E\left\{d_{n}^{2} \mid \mathcal{F}_{n-1}\right\}+\frac{\lambda^{3}}{3 !} E\left\{d_{n}^{3} \mid \mathcal{F}_{n-1}\right\}+\cdots \\
& \leq 1+\frac{\lambda^{2} b^{2}}{2}\left(1+\frac{\lambda c}{3}+\frac{\lambda^{2} c^{2}}{4.3}+\cdots\right) \\
& \leq \exp \left(\frac{\lambda^{2}}{2} \cdot b^{2} e^{\lambda c}\right)
\end{aligned}
$$

since $1+t / 3+t^{2} / 4.3+\cdots \leq e^{t}$ and $1+t \leq e^{t}(t>0)$. The conclusion to the lemma is then obtained from a simple iteration.

The estimates of the differences required to apply this exponential inequality are drawn from Lemma 2.1, whose bounds, however, involve the partial sum and therefore the martingale in consideration. This difficulty is overcome using an iteration procedure borrowed from [8]. This and the different steps of the proof of the main result will now be made precise in the next section.

3. The main result. Recall that a Banach space $B$ is superreflexive if every Banach space finitely representable in $B$ is reflexive and that any superreflexive space admits an equivalent uniformly convex norm. The main result of this paper is the following theorem. 
THEOREM 3.1. Let $B$ be a superreflexive Banach space. A random variable $X$ with values in $B$ satisfies the bounded LIL iff the following three conditions are fulfilled:

(3.1) $E\left\{\|X\|^{2} / L_{2}\|X\|\right\}<\infty$;

(3.2) for each $x^{*}$ in $B^{*}, E\left\{\left|x^{*}(X)\right|^{2}\right\}<\infty$;

(3.3) the sequence $\left(S_{n} / a_{n}\right)_{n \in \mathbf{N}}$ is stochastically bounded.

COROLlaRY 3.2. Let $B$ be a superreflexive Banach space. A random variable $X$ with values in $B$ satisfies the compact LIL iff (3.1) holds and

(3.4) the family of real random variables $\left\{\left|x^{*}(X)\right|^{2} ; x^{*} \in B^{*},\left\|x^{*}\right\|=1\right\}$ is uniformly integrable;

(3.5) $S_{n} / a_{n} \rightarrow 0$ in probability.

CoRollary 3.3. Let $B$ be a superreflexive type 2 Banach space. A mean zero random variable $X$ with values in $B$ satisfies the bounded (resp. compact) LIL iff (3.1) and (3.2) (resp. (3.4)) hold.

As a last corollary to Theorem 3.1, we mention the following one which does not seem to follow from the relation between the CLT and LIL, implying only that the bounded LIL holds.

COROLLARY 3.4. Let $B$ be a superreflexive Banach space, and let $X$ be a random variable with values in $B$ such that the sequence $\left(S_{n} / \sqrt{n}\right)_{n \in \mathbf{N}}$ is stochastically bounded; then $X$ satisfies the compact LIL iff $E\left\{\|X\|^{2} / L_{2}\|X\|\right\}<\infty$.

Before turning to the proof of Theorem 3.1, let us make some comments on the previous corollaries and indicate how they can be deduced from Theorem 3.1. Note first that Theorem 3.1 and Corollary 3.2 characterize the LIL in $L_{p}$ spaces for $1<p<\infty$. The cases $p=1$ and $p=\infty$ are therefore still open; as shown by M. Klass and J. Kuelbs [13], the equivalence of Theorem 3.1 holds for random variables in $l_{1}$ or $l_{\infty}$ with independent coordinates. Corollary 3.3 follows immediately from Theorem 3.1 and Corollary 3.2 since in type 2 spaces, as already pointed out in the introduction, (3.5) is satisfied under (3.1) and $E\{X\}=0$ [6, Proposition 7.2]. Note that type 2 spaces need not be reflexive [12]; Corollary 3.3 improves upon the main result of [18] since, for each $1<p \leq 2$, there is a superreflexive type $p$ Banach space which is not $p$-smoothable [24]. Corollary 3.4 is a simple consequence of Corollary 3.2 since random variables with $\left(S_{n} / \sqrt{n}\right)_{n \in \mathbf{N}}$ stochastically bounded are pregaussian (and therefore satisfy (3.4) in spaces which do not contain an isomorphic copy of $c_{0}[\mathbf{2 6}]$, in particular uniformly convex spaces.

Let us now prove Corollary 3.2. We first provide a simple proof of the necessity of (3.4); that (3.5) is necessary follows from the fact that if $X$ satisfies the compact LIL, the sequence of laws of the $S_{n} / a_{n}$ is tight with only 0 as limit point. Suppose the family of random variables $\left\{\left|x^{*}(X)\right|^{2} ;|| x^{*}||=1\right\}$ is not uniformly integrable; then there is an $\varepsilon>0$ such that

$$
\limsup _{c \rightarrow \infty} \sup _{\| x^{*} \mid=1} \int_{\left\{\left|x^{*}(X)\right|>c\right\}}\left|x^{*}(X)\right|^{2} d P>\varepsilon .
$$

Therefore, one can construct a sequence $\left(c_{k}\right)$ of real numbers increasing to infinity and a family $\left(x_{k}^{*}\right)$ of linear functionals of norm one such that, for each $k$,

$$
\int_{\left\{\|X\|>c_{k}\right\}}\left|x_{k}^{*}(X)\right|^{2} d P \geq \int_{\left\{\left|x_{k}^{*}(X)\right|>c_{k}\right\}}\left|x_{k}^{*}(X)\right|^{2} d P>\varepsilon .
$$


Let $\left(x_{k^{\prime}}^{*}\right)$ be a weakly convergent subsequence of $\left(x_{k}^{*}\right)$ converging to some $x^{*}$. By compactness,

$$
\lim _{k^{\prime} \rightarrow \infty} \sup _{n}\left|\left(x_{k^{\prime}}^{*}-x^{*}\right)\left(\frac{S_{n}}{a_{n}}\right)\right|=0 \quad \text { w.p.1. }
$$

The scalar LIL then implies that

$$
\lim _{k^{\prime} \rightarrow \infty} E\left\{\left|\left(x_{k^{\prime}}^{*}-x^{*}\right)(X)\right|^{2}\right\}=0
$$

which clearly contradicts our first claim and thus establishes the necessity of (3.4). Note that this proof shows that (3.4) is satisfied iff the covariance function $T\left(x^{*}, y^{*}\right)$ $=E\left\{x^{*}(X) y^{*}(X)\right\}, x^{*}, y^{*} \in B^{*}$, is weakly sequentially continuous. It is, moreover, equivalent to the compactness of the covariance operator (or the unit ball $K$ of the reproducing kernel Hilbert space) of $X$ (see [6]).

To complete the proof of Corollary 3.2, we have to show the sufficiency. We first need to recall some integrability results. Using the technique of Proposition 2.1 of $[\mathbf{2 5}],(3.3)$ is clearly seen to be equivalent to

$$
\forall \alpha \in(0,2), \quad R_{\alpha}(X)=\sup _{n}\left(E\left\{\left(\frac{\left\|S_{n}\right\|}{a_{n}}\right)^{\alpha}\right\}\right)^{1 / \alpha}<\infty .
$$

If the sequence $\left(S_{n} / a_{n}\right)_{n \in \mathbf{N}}$ is almost surely bounded (i.e., $X$ satisfies the bounded LIL), then (3.1) and the integrability theorems of [9] show that

$$
\forall \alpha \in(0,2), \quad \mathrm{IL}_{\alpha}(X)=\left(E\left\{\sup _{n}\left(\frac{\left\|S_{n}\right\|}{a_{n}}\right)^{\alpha}\right\}\right)^{1 / \alpha}<\infty .
$$

In the following, we use the notation $R$ and IL for $R_{1}$ and $\mathrm{IL}_{1}$.

Having recalled these properties, let $\Phi(t)=t^{2} / L_{2} t, t \geq 0$, and

$$
\|X\|_{\Phi}=\inf \{a>0: E\{\Phi(\|X\| / a)\} \leq 1\},
$$

which defines a norm on the Orlicz space of all $B$-valued random variables $X$ satisfying (3.1). By a closed graph argument, Theorem 3.1 implies the existence of a positive constant $C$ such that for every random variable $X$ with values in $B$,

$$
\mathrm{IL}(X) \leq C\left(\|X\|_{\Phi}+\sigma(X)+R(X)\right) .
$$

Given $X$ satisfying (3.1), (3.4) and (3.5), consider an increasing family $\left(\mathcal{F}^{N}\right)_{N \in \mathbf{N}}$ of finite $\sigma$-fields generating the $\sigma$-field of $X$ and let $X^{N}=E\left\{X \mid \xi^{N}\right\}$. It is easily seen that

$$
\lim _{N \rightarrow \infty}\left(\left\|X-X^{N}\right\|_{\Phi}+\sigma\left(X-X^{N}\right)+R\left(X-X^{N}\right)\right)=0 .
$$

Indeed, that $\lim _{N \rightarrow \infty}\left\|X-X^{N}\right\|_{\Phi}=0$ follows from the martingale convergence theorem. Under (3.4), Jensen's inequality implies that the family $\left\{\left|x^{*}\left(X-X^{N}\right)\right|^{2}\right.$; $\left.\left\|x^{*}\right\|=1, N \in \mathbf{N}\right\}$ is uniformly integrable; therefore, for each $\varepsilon>0$, there is a $\delta>0$ such that for all measurable sets $A$ with $P(A)<\delta$,

$$
\sup _{\left\|x^{*}\right\|=1} \sup _{N} \int_{A}\left|x^{*}\left(X-X^{N}\right)\right|^{2} d P<\varepsilon^{2} .
$$

For $N$ large enough, $P\left\{\left\|X-X^{N}\right\|>\varepsilon\right\}<\delta$ so that

$$
\sigma^{2}\left(X-X^{N}\right) \leq \sup _{\left\|x^{*}\right\|=1} \int_{\left\{\left\|X-X^{N}\right\| \leq \varepsilon\right\}}\left|x^{*}\left(X-X^{N}\right)\right|^{2} d P+\varepsilon^{2} \leq 2 \varepsilon^{2} .
$$


Hence $\lim _{N \rightarrow \infty} \sigma\left(X-X^{N}\right)=0$. Finally, remembering (3.6), if $S_{n} / a_{n} \rightarrow 0$ in probability, this convergence also takes place in $L_{1}$ so that, given $\varepsilon>0$, for all $n$ large enough and all $N$,

$$
E\left\{\left\|S_{n}-S_{n}^{N}\right\| / a_{n}\right\} \leq 2 E\left\{\left\|S_{n}\right\| / a_{n}\right\}<\varepsilon \quad\left(S_{n}^{N}=X_{1}^{N}+\cdots+X_{n}^{N}\right),
$$

which easily implies that $\lim _{N \rightarrow \infty} R\left(X-X^{N}\right)=0$. Applying (3.7) to $X-X^{N}$ for each $N$, we have thus obtained that

$$
\lim _{N \rightarrow \infty} \operatorname{IL}\left(X-X^{N}\right)=0
$$

the random variables $X^{N}$ being finite valued, this finite dimensional approximation clearly establishes that $X$ satisfies the compact LIL.

We now prove Theorem 3.1.

PROOF OF THEOREM 3.1. Note first that the expected characterization of the LIL through conditions (3.1), (3.2), (3.3) is invariant under isomorphism. Since $B$ is superreflexive, it admits an equivalent $p$-smooth norm for some $p>1$; we equip $B$ with this $p$-smooth norm, again denoted $\|\cdot\|$, for which formula (2.2) and Lemma $2.1 \mathrm{apply}$, and we will prove the equivalence of the theorem for this norm. We assume $p<2$, although the proof presented below can easily be adapted to the case $p=2$ and is actually simpler; this case was settled in $[\mathbf{1 8}]$.

We need only show the sufficiency of conditions (3.1), (3.2) and (3.3). Let $X$ satisfy these conditions. Since by centering and Jensen's inequality,

$$
\operatorname{IL}(X) \leq 2 \operatorname{IL}(\varepsilon X) \text { and } R(\varepsilon X) \leq 2 R(X),
$$

where $\varepsilon$ denotes a Rademacher random variable independent of $X$, we may and do assume $X$ to be symmetric. We may also suppose without any loss of generality that

$$
E\left\{\|X\|^{2} / L_{2}\|X\|\right\} \leq 1, \quad \sigma=\sigma(X) \leq 1 \quad \text { and } \quad R_{p}(X) \leq \frac{1}{2} .
$$

For each integer $k$, let $I(k)=\left\{2^{k}+1, \ldots, 2^{k+1}\right\}$ and set, for all $k$ and $i$ in $I(k)$,

$$
\begin{aligned}
& u_{i}=X_{i} I_{\left\{\left\|X_{i}\right\|^{2} \leq 2^{k} /\left(L_{2} k\right)^{\alpha}\right\}}, \quad U_{k}=\sum_{i \in I(k)} u_{i} ; \\
& \left.v_{i}=X_{i} I_{\left\{2^{k} /\left(L_{2} k\right)^{\alpha}\right.}<\left\|X_{i}\right\|^{2} \leq a_{2^{k}}^{2}\right\}, \quad V_{k}=\sum_{i \in I(k)} v_{i} ; \\
& w_{i}=X_{i} I_{\left\{\left\|X_{i}\right\|^{2}>a_{2^{k}}{ }^{k}\right\}}, \quad W_{k}=\sum_{i \in I(k)} w_{i},
\end{aligned}
$$

where $\alpha=\left[(p-1)^{2}+1\right] /(2-p)(p-1)$. Let us note for what follows that

$$
\alpha>[(2-p)(p-1)]^{-1}>(p-1)^{-1}>1,
$$

so that in all cases $\alpha+1>(p-1)^{-1}$ and $\alpha>1$. (When $p=2$, it is possible to take $\alpha=0$ in the proof below.) By symmetry and a classical blocking argument (see $[\mathbf{2 7}$, p. 159]), for $X$ to satisfy the bounded LIL it is sufficient to show that with probability one,

$$
\sup _{k} \frac{\left\|U_{k}\right\|}{a_{2^{k}}}<\infty
$$




$$
\begin{aligned}
& \sup _{k} \frac{\left\|V_{k}\right\|}{a_{2^{k}}}<\infty, \\
& \lim _{k} \frac{\left\|W_{k}\right\|}{a_{2^{k}}}=0 .
\end{aligned}
$$

That (3.11) is satisfied follows immediately from the Borel-Cantelli lemma, since for every $\varepsilon>0$,

$$
\sum_{k} P\left\{\left\|W_{k}\right\|>\varepsilon a_{2^{k}}\right\} \leq \sum_{k} 2^{k} P\left\{\|X\|>a_{2^{k}}\right\},
$$

which is finite under (3.1). Let us show (3.10).

LEMMA 3.5. Under (3.8),

$$
\sup _{k} \frac{\left\|V_{k}\right\|}{a_{2^{k}}}<\infty \quad \text { w.p.1. }
$$

ProOF. Since $R_{p}(X) \leq \frac{1}{2}$, by symmetry and a contraction principle (cf. [9])

$$
E\left\{\left\|V_{k}\right\|^{p}\right\} \leq a_{2^{k}}^{p} \quad \text { for all } k .
$$

Let $r=p / 2(p-1)>1$. To establish (3.10), since $\left\|v_{i}\right\| \leq a_{2^{k}}$ for $i \in I(k)$, the BorelCantelli lemma and J. Hoffmann-Jørgensen's inequality [9, p. 164, (3.3)] iterated $m$ times, where $2^{m} \geq r$, show that it is enough to prove that

$$
\sum_{k}\left(P\left\{\left\|V_{k}\right\|^{p}>2 a_{2^{k}}^{p}\right\}\right)^{r}<\infty .
$$

Using (3.12) and Lemma 2.1, for all $k$

$$
\begin{aligned}
P\left\{\left\|V_{k}\right\|^{p}>2 a_{2^{k}}^{p}\right\} & \leq P\left\{\left\|V_{k}\right\|^{p}-E\left\{\left\|V_{k}\right\|^{p}\right\}>a_{2^{k}}^{p}\right\} \\
& \leq 2 a_{2^{k}}^{-2 p}\left(p^{2} \sum_{i \in I(k)} E\left\{F_{p}^{2}\left(V_{k}-v_{i}\right)\left(v_{i}\right)\right\}+C^{2} \sum_{i \in I(k)} E\left\{\left\|v_{i}\right\|^{2 p}\right\}\right) .
\end{aligned}
$$

Letting $K$ denote a positive finite constant possibly changing from line to line, it is easily seen that

$$
\begin{aligned}
& {\left[\sum_{k}\left(a_{2^{k}}^{-2 p} \sum_{i \in I(k)} E\left\{\left\|v_{i}\right\|^{2 p}\right\}\right)^{r}\right]^{1 / r}} \\
& \quad \leq \sum_{k} a_{2^{k}}^{-2 p} \cdot 2^{k} E\left\{\|X\|^{2 p} I_{\left\{\|X\| \leq a_{2^{k}}\right\}}\right\} \\
& \quad \leq K E\left\{\|X\|^{2 p}\left(L_{2}\|X\|\right)^{-p}\left(\|X\|^{2} / L_{2}\|X\|\right)^{1-p}\right\} \\
& \quad \leq K E\left\{\|X\|^{2} / L_{2}\|X\|\right\}<\infty .
\end{aligned}
$$

On the other hand, by independence and Fubini's theorem, for every $k$ and $i \in I(k)$,

$$
\begin{aligned}
E\left\{F_{p}^{2}\left(V_{k}-v_{i}\right)\left(v_{i}\right)\right\} & =E\left\{\left\|V_{k}-v_{i}\right\|^{2(p-1)} \frac{F_{p}^{2}\left(V_{k}-v_{i}\right)}{\left\|F_{p}\left(V_{k}-v_{i}\right)\right\|^{2}}\left(v_{i}\right)\right\} \\
& \leq \sigma^{2} E\left\{\left\|V_{k}-v_{i}\right\|^{2(p-1)}\right\} \leq E\left\{\left\|V_{k}-v_{i}\right\|^{2(p-1)}\right\} .
\end{aligned}
$$


Further, Jensen's inequality, the previous inequality, and the type $p$ property of $p$-smooth norms (with constant $C$ as in (2.1) for this one) imply that, for all $i$ in $I(k)$,

$$
E\left\{F_{p}^{2}\left(V_{k}-v_{i}\right)\left(v_{i}\right)\right\} \leq\left(E\left\{\left\|V_{k}-v_{i}\right\|^{p}\right\}\right)^{1 / r} \leq\left(C \cdot 2^{k} E\left\{\left\|v_{i}\right\|^{p}\right\}\right)^{1 / r} .
$$

Hence

$$
\begin{aligned}
& \sum_{k} a_{2^{k}}^{-2 p r}\left(\sum_{i \in I(k)} E\left\{F_{p}^{2}\left(V_{k}-v_{i}\right)\left(v_{i}\right)\right\}\right)^{r} \\
& \quad \leq K \sum_{k} 2^{k(1-p / 2)}\left(L_{2} 2^{k}\right)^{-p r} E\left\{\|X\|^{p} I_{\left\{\|X\|^{2} \geq 2^{k} /\left(L_{2} 2^{k}\right)^{\alpha}\right\}}\right\} \\
& \quad \leq K E\left\{\|X\|^{p}\left(L_{2}\|X\|\right)^{-p r}\left[\|X\|^{2}\left(L_{2}\|X\|\right)^{\alpha}\right]^{1-p / 2}\right\} \\
& \quad=K E\left\{\|X\|^{2} / L^{2}\|X\|\right\}<\infty,
\end{aligned}
$$

since $\alpha(1-p / 2)=p r-1$. (3.14) and (3.15) therefore imply (3.13) and thus the conclusion of the lemma.

We now turn to the main step of the proof, which consists in showing (3.9). As we will repeatedly use Lemma 2.1 for $U_{k}=\sum_{i \in I(k)} u_{i}$, let us specify the notation in this regard. We set, for each $k$,

$$
\left\|U_{k}\right\|^{p}-E\left\{\left\|U_{k}\right\|^{p}\right\}=\sum_{i \in I(k)} d_{i},
$$

where $d_{i}=E\left\{\| U_{k}||^{p} \mid \mathcal{F}_{i}\right\}-E\left\{\left\|U_{k}\right\|^{p} \mid \mathcal{F}_{i-1}\right\}, \mathcal{F}_{i}$ the $\sigma$-field generated by $X_{2^{k}+1}, \ldots$, $X_{i}, \mathcal{F}_{2^{k}}$ the trivial field. The martingale differences $d_{i}$ and the $\sigma$-fields $\mathcal{F}_{i}$ of course depend on $k$, but we suppress this.

Let $M$ be a positive numerical constant to be specified later, and let $t$ be any real number $\geq 1$. Again by contraction and (3.8),

$$
E\left\{\left\|U_{k}\right\|^{p}\right\} \leq a_{2^{k}}^{p} \quad \text { for each } k .
$$

We estimate, for each $k$,

$$
\begin{aligned}
P\left\{\left\|U_{k}\right\|^{p}>(M t+1) a_{2^{k}}^{p}\right\} \leq & P\left\{\left\|U_{k}\right\|^{p}-E\left\{\left\|U_{k}\right\|^{p}\right\}>M t a_{2^{k}}^{p}\right\} \\
\leq & P\left\{\sup _{i \in I(k)} \sum_{j=2^{k}+1}^{i} d_{j}>M t a_{2^{k}}^{p}\right\} \\
\leq & P\left\{\sup _{i \in I(k)} \sum_{j=2^{k}+1}^{i} \tilde{d}_{j}>M t a_{2^{k}}^{p}\right\} \\
& +P\left\{\sup _{i \in I(k)} E\left\{\left\|U_{k}\right\|^{p} \mid \mathcal{F}_{i-1}\right\}>(M+1) t^{2} a_{2^{k}}^{p}\right\},
\end{aligned}
$$

where $\tilde{d}_{i}=d_{i} I_{\left\{E\left\{|| U_{k}|| p \mid \mathcal{F}_{i-1}\right\} \leq(M+1) t^{2} a_{2^{k}}^{p}\right\}}, i \in I(k)$, which define a new martingale differences sequence. The next lemma will follow as an application of Lemmas 2.1 and 2.2 . 
LEMMA 3.6. There exists an integer $k_{0}$ such that for $k \geq k_{0}, M=50$ (say) and any $t \geq 1$,

$$
P\left\{\sup _{i \in I(k)} \sum_{j=2^{k}+1}^{i} \tilde{d}_{j}>M t a_{2^{k}}^{p}\right\} \leq \exp \left(-2 L_{2} 2^{k}\right) .
$$

PROOF. Let $t \geq 1$ be fixed. We apply the exponential inequality of Lemma 2.2 to the martingale differences sequences $\left(\tilde{d}_{i}, \mathcal{F}_{i}\right)_{i \in I(k)}$ for each $k$; we get, $\lambda>0$,

$$
P\left\{\sup _{i \in I(k)} \sum_{j=2^{k}+1}^{i} \tilde{d}_{j}>M t a_{2^{k}}^{p}\right\} \leq \exp \left(-\lambda M t a_{2^{k}}^{p}+\frac{\lambda^{2}}{2} \cdot 2^{k} b^{2} e^{\lambda c}\right),
$$

where $b, c>0$ are determined in the following way: according to Lemma 2.1, for each $i$ in $I(k)$,

$$
E\left\{d_{i}^{2} \mid \mathcal{F}_{i-1}\right\} \leq 2 p^{2} E\left\{F_{p}^{2}\left(U_{k}-u_{i}\right)\left(u_{i}\right) \mid \mathcal{F}_{i-1}\right\}+2 C^{2} E\left\{\left\|u_{i}\right\|^{2 p}\right\} .
$$

As in Lemma 3.5, by independence and Jensen's inequality $(r=p / 2(p-1)>1$ and $X$ is symmetric)

$$
\begin{aligned}
E\left\{F_{p}^{2}\left(U_{k}-u_{i}\right)\left(u_{i}\right) \mid \mathcal{F}_{i-1}\right\} & \leq \sigma^{2} E\left\{|| U_{k}-u_{i} \|^{2(p-1)} \mid \mathcal{F}_{i-1}\right\} \\
& \leq\left(E\left\{\left\|U_{k}-u_{i}\right\|^{p} \mid \mathcal{F}_{i-1}\right\}\right)^{1 / r} \leq\left(E\left\{\left\|U_{k}\right\|^{p} \mid \mathcal{F}_{i-1}\right\}\right)^{1 / r}
\end{aligned}
$$

thus

$$
\begin{aligned}
E\left\{\tilde{d}_{i}^{2} \mid \mathcal{F}_{i-1}\right\} & \leq 8\left[(M+1) t^{2}\right]^{1 / r} a_{2^{k}}^{2(p-1)}+2 C^{2} E\left\{\left\|u_{i}\right\|^{2 p}\right\} \\
& \leq 8(M+1) t^{2} a_{2^{k}}^{2(p-1)}+2 C^{2} E\left\{\|X\|^{2 p} I_{\left\{\|X\|^{2} \leq 2^{k} /\left(L_{2} 2^{k}\right)^{\alpha}\right\}}\right\} \\
& \leq 8(M+1) t^{2} a_{2^{k}}^{2(p-1)}+2 C^{2} 2^{k(p-1)}\left(L_{2} 2^{k}\right)^{1-\alpha(p-1)},
\end{aligned}
$$

since $E\left\{\|X\|^{2} / L_{2}\|X\|\right\} \leq 1$. The choice of $\alpha\left(\alpha+1>(p-1)^{-1}\right)$ implies that

$$
2^{k(p-1)}\left(L_{2} 2^{k}\right)^{1-\alpha(p-1)}=o\left(a_{2^{k}}^{2(p-1)}\right)
$$

hence, for $k$ large enough and $i$ in $I(k)$,

$$
E\left\{\tilde{d}_{i}^{2} \mid \mathcal{F}_{i-1}\right\} \leq 9(M+1) t^{2} a_{2^{k}}^{2(p-1)}=b^{2} .
$$

Concerning $c$, Lemma 2.1 and Jensen's inequality again imply that for each $i \in I(k)$ and all $k$,

$$
\begin{aligned}
\left|d_{i}\right| & \leq p\left(\left\|u_{i}\right\|+E\left\{\left\|u_{i}\right\|\right\}\right) E\left\{\left\|U_{k}-u_{i}\right\|^{p-1} \mid \mathcal{F}_{i-1}\right\}+C\left(\left\|u_{i}\right\|^{p}+E\left\{\left\|u_{i}\right\|^{p}\right\}\right) \\
& \leq p\left(\left\|u_{i}\right\|+E\left\{\left\|u_{i}\right\|\right\}\right)\left(E\left\{\left\|U_{k}\right\|^{p} \mid \mathcal{F}_{i-1}\right\}\right)^{(p-1) / p}+C\left(\left\|u_{i}\right\|^{p}+E\left\{\left\|u_{i}\right\|^{p}\right\}\right),
\end{aligned}
$$

so that

$$
\begin{aligned}
\left|\tilde{d}_{i}\right| & \leq 2 p 2^{k / 2}\left(L_{2} 2^{k}\right)^{-\alpha / 2}\left[(M+1) t^{2} a_{2^{k}}^{p}\right]^{(p-1) / p}+2 C 2^{k p / 2}\left(L_{2} 2^{k}\right)^{-\alpha p / 2} \\
& \leq(M+1)^{1 / 2} t a_{2^{k}}^{p} / L_{2} 2^{k}=c
\end{aligned}
$$

for $k$ sufficiently large since $2(p-1) \leq p$ and $\alpha>1$. Therefore, for $k$ larger than some $k_{0}$, the exponential inequality (3.18) holds for all $\lambda>0$ for the choice of $b$, $c>0$ as obtained in (3.19) and (3.20); that is, for any $k \geq k_{0}$ and $\lambda>0$,

$$
\begin{aligned}
P\left\{\sup _{i \in I(k)} \sum_{j=2^{k}+1}^{i} \tilde{d}_{j}>M t a_{2^{k}}^{p}\right\} \\
\leq \exp \left(-\lambda M t a_{2^{k}}^{p}+\left(\lambda^{2} / 2\right) \cdot 2^{k} 9(M+1) t^{2} a_{2^{k}}^{2(p-1)} e^{\lambda c}\right) .
\end{aligned}
$$


Setting $\lambda=3 L_{2} 2^{k} / M t a_{2^{k}}^{p}$ for $k \geq k_{0}$, we get

$$
\begin{aligned}
& P\left\{\sup _{i \in I(k)} \sum_{j=2^{k}+1}^{i} \tilde{d}_{j}>M t a_{2^{k}}^{p}\right\} \\
& \quad \leq \exp \left[-L_{2} 2^{k}\left(3-\frac{81}{4} \cdot \frac{M+1}{M^{2}} \cdot e^{3(M+1)^{1 / 2} / M}\right)\right] .
\end{aligned}
$$

The choice of $M=50$, for example, then clearly implies the lemma.

(3.16) and (3.17) now imply that for all $k$ large enough, $M=50$ and $t \geq 1$,

$$
\begin{aligned}
& P\left\{\sup _{i \in I(k)} \sum_{j=2^{k}+1}^{i} d_{j}>M t a_{2^{k}}^{p}\right\} \\
& \quad \leq P\left\{\sup _{i \in I(k)} \sum_{j=2^{k}+1}^{i} d_{j}>M t^{2} a_{2^{k}}^{p}\right\}+\exp \left(-2 L_{2} 2^{k}\right) .
\end{aligned}
$$

Indeed, for each $i$ in $I(k)$,

$$
\begin{aligned}
E\left\{\left\|U_{k}\right\|^{p} \mid \mathcal{F}_{i-1}\right\} & =E\left\{\left\|U_{k}\right\|^{p}-E\left\{\left\|U_{k}\right\|^{p}\right\} \mid \mathcal{F}_{i-1}\right\}+E\left\{\left\|U_{k}\right\|^{p}\right\} \\
& =\sum_{j=2^{k}+1}^{i-1} d_{j}+E\left\{\left\|U_{k}\right\|^{p}\right\} \leq \sup _{i \in I(k)} \sum_{j=2^{k}+1}^{i} d_{j}+a_{2^{k}}^{p}
\end{aligned}
$$

since $E\left\{\left\|U_{k}\right\|^{p}\right\} \leq a_{2^{k}}^{p}$ for all $k$, and therefore, $t$ being $\geq 1$,

$$
\begin{gathered}
P\left\{\sup _{i \in I(k)} E\left\{\left\|U_{k}\right\|^{p} \mid \mathcal{F}_{i-1}\right\}>(M+1) t^{2} a_{2^{k}}^{p}\right\} \\
\leq P\left\{\sup _{i \in I(k)} \sum_{j=2^{k}+1}^{i} d_{j}>M t^{2} a_{2^{k}}^{p}\right\} .
\end{gathered}
$$

Formula (3.21) (with $M=50$ ) is valid for all $k$ larger than some $k_{0}$ and any real $t \geq 1$. Iterating it $N$ times, we get

$$
\begin{aligned}
& P\left\{\sup _{i \in I(k)} \sum_{j=2^{k}+1}^{i} d_{j}>M t a_{2^{k}}^{p}\right\} \\
& \quad \leq P\left\{\sup _{i \in I(k)} \sum_{j=2^{k}+1}^{i} d_{j}>M t^{2^{N}} a_{2^{k}}^{p}\right\}+N \exp \left(-2 L_{2} 2^{k}\right)
\end{aligned}
$$

for all $k \geq k_{0}$. Taking $t$ to be 2 and $N$ the integer part of $(L k / L 2+1)$ for $k \geq k_{0}$, and recalling (3.16), the proof of (3.9) will be completed using the Borel-Cantelli lemma if

$$
\sum_{k} P\left\{\sup _{i \in I(k)} \sum_{j=2^{k}+1}^{i} d_{j}>M 2^{k} a_{2^{k}}^{p}\right\}<\infty,
$$


which is actually immediate since the martingale maximal inequality implies that

$$
P\left\{\sup _{i \in I(k)} \sum_{j=2^{k}+1}^{i} d_{j}>M 2^{k} a_{2^{k}}^{p}\right\} \leq \frac{2 E\left\{\left\|U_{k}\right\|^{p}\right\}}{M 2^{k} a_{2^{k}}^{p}} \leq \frac{2}{M 2^{k}} .
$$

This thus shows that (3.9) holds under the conditions of Theorem 3.1 and therefore completes its proof.

4. A general property. If $0<p, q<\infty$, denote by $L_{p, q}$ the Lorentz space of all real random variables $\xi$ such that

$$
\|\xi\|_{p, q}=\left(q \int_{0}^{\infty}\left(t^{p} P\{|\xi|>t\}\right)^{q / p} \frac{d t}{t}\right)^{1 / q}<\infty .
$$

$L_{p, p}$ is just $L_{p}$ by the usual integration by parts formula; $L_{p, q} \subseteq L_{p, q^{\prime}}$, if $q \leq q^{\prime}$.

If $X$ is a Banach space valued random variable satisfying the CLT and $\xi$ a real random variable belonging to $L_{2,1}$ and independent of $X$, then following [5, Lemma 2.9 ], the product $\xi X$ also satisfies the CLT, the integrability condition on $\xi$ being the best possible in general $[\mathbf{2 0}]$.

We have seen that conditions (3.1), (3.2) and (3.3) (resp. (3.1), (3.4) and (3.5)) are expected to be necessary and sufficient for a Banach space valued random variable $X$ to satisfy the bounded (resp. compact) LIL, and we have proved such an equivalence in uniformly convex Banach spaces. If such an equivalence should hold in any Banach space, the multiplication property described before for the CLT should apply in the same way to the LIL since the stochastic boundedness or convergence to 0 of the sequence $\left(S_{n} / a_{n}\right)_{n \in \mathbf{N}}$ behaves like the CLT with respect to such a property. We can actually prove directly that this is indeed the case.

THEOREM 4.1. Let $B$ be a Banach space, $X$ a B-valued random variable and $\xi$ a real random variable independent of $X$; if $\xi$ is in $L_{2,1}$ and $X$ satisfies the bounded (resp. compact) LIL, so does the product $\xi X$. Moreover, the integrability condition on $\xi$ cannot be improved in general.

PROOF. It is enough to show the existence of a positive constant $C$ such that for every random variable $X$ with values in $B$,

$$
\operatorname{IL}(\xi X) \leq C\|\xi\|_{2,1} \operatorname{IL}(X) .
$$

This clearly settles the bounded LIL case; if $X$ satisfies the compact LIL, the approximation property of Théorème 3.1 of $[\mathbf{2 5}]$ implies the existence, for each $\varepsilon>0$, of a step random variable $Y$ such that $\operatorname{IL}(X-Y)<\varepsilon$, so that (4.1) applied to $X-Y$ yields

$$
\operatorname{IL}(\xi(X-Y))<C\|\xi\|_{2,1} \varepsilon
$$

and therefore that $\xi X$ also satisfies the compact LIL.

Let us now prove (4.1). Denote by $\left(\xi_{n}\right)_{n \in \mathbf{N}}$ a sequence of independent copies of $\xi$ (independent of $\left.\left(X_{n}\right)_{n \in \mathbf{N}}\right)$ and let, for every $n, S_{n}(\xi)=\xi_{1}+\cdots+\xi_{n}$ and $S_{n}(\xi X)=\xi_{1} X_{1}+\cdots+\xi_{n} X_{n}$. Suppose first $\xi$ is the indicator function of some measurable set $A$. Checking Fourier transforms, it is easily seen that the sequences 
$\left(S_{n}(\xi X)\right)_{n \in \mathbf{N}}$ and $\left(\sum_{i=1}^{S_{n}(\xi)} X_{i}\right)_{n \in \mathbf{N}}$ have the same distribution. Hence

$$
\begin{aligned}
\operatorname{IL}(\xi X) & =E\left\{\sup _{n} \frac{\left\|\sum_{i=1}^{S_{n}(\xi)} X_{i}\right\|}{a_{n}}\right\} \\
& =E\left\{\sup _{n}\left(\frac{S_{n}(\xi) L_{2} S_{n}(\xi)}{n L_{2} n}\right)^{1 / 2} \frac{\left\|\sum_{i=1}^{S_{n}(\xi)} X_{i}\right\|}{a_{S_{n}}(\xi)}\right\}
\end{aligned}
$$

and therefore by independence of $X$ and $\xi$,

$$
\begin{aligned}
\mathrm{IL}(\xi X) & \leq E\left\{\sup _{n}\left(\frac{S_{n}(\xi) L_{2} S_{n}(\xi)}{n L_{2} n}\right)^{1 / 2}\right\} \mathrm{IL}(X) \\
& \leq E\left\{\sup _{n}\left(\frac{S_{n}(\xi)}{n}\right)^{1 / 2}\right\} \mathrm{IL}(X) .
\end{aligned}
$$

Now if $\xi$ is any real integrable random variable, the strong law of large numbers and the integrability theorems of J. Hoffmann-Jørgensen [9] indicate that

$$
E\left\{\sup _{n}\left|\frac{S_{n}(\xi)}{n}\right|^{p}\right\}<\infty \quad \text { for any } 0<p<1 .
$$

In fact, a closed graph argument or an elementary computation yield the existence of a positive constant $C$ depending only upon $p$ such that for all $\xi$,

$$
E\left\{\sup _{n}\left|\frac{S_{n}(\xi)}{n}\right|^{p}\right\} \leq C(E\{|\xi|\})^{p} .
$$

Therefore, if $\xi=I_{A}$, we have by (4.2)

$$
\mathrm{IL}(\xi X) \leq C(P(A))^{1 / 2} \mathrm{IL}(X) .
$$

Now let $\xi$ be positive in $L_{2,1}$, independent of $X$, and set, for each $\varepsilon>0, \xi_{\varepsilon}=$ $\sum_{k=1}^{\infty} \varepsilon I_{\{\xi>\varepsilon k\}}$ so that $\lim _{\varepsilon \rightarrow 0} \xi_{\varepsilon}=\xi$ with probability one. Clearly

$$
\sum_{k=1}^{\infty} \varepsilon(P\{\xi>\varepsilon k\})^{1 / 2} \leq\|\xi\|_{2,1}
$$

and by the triangle inequality and (4.3)

$$
\mathrm{IL}\left(\xi_{\varepsilon} X\right) \leq \sum_{k=1}^{\infty} \varepsilon \mathrm{IL}\left(I_{\{\xi>\varepsilon k\}} X\right) \leq C\|\xi\|_{2,1} \operatorname{IL}(X) .
$$

Letting $\varepsilon$ go to 0 then implies (4.1) and the first part of Theorem 4.1. That the integrability condition on $\xi$ is the best possible follows from straightforward modifications of the same property for the CLT presented in [20]: the finite dimensional norm on $\mathbf{R}^{q}$ of the first step in [20] has only to be changed to

$$
\|x\|_{q, m}=\sup \left\{\sum_{j=1}^{m}\left(\frac{L_{2} j}{j}\right)^{1 / 2}\left|x_{i_{j}}\right| ;\left\{i_{1}, \ldots, i_{m}\right\} \subset\{1, \ldots, q\}\right\},
$$

where $x=\left(x_{i}\right)_{i \leq q}$ and $m \leq q$. The construction of [20] then implies the existence for any $\xi$ not in $L_{2,1}$ of a bounded Banach space valued random variable $X$ independent of $\xi$ such that $S_{n} / a_{n} \rightarrow 0$ in probability (thus satisfying the compact LIL by [17]) but for which the sequence $\left(S_{n}(\xi X) / a_{n}\right)_{n \in \mathbf{N}}$ is not stochastically bounded.

ADDED IN PROOF. Since this paper was submitted, I have proved that Corollary 3.3 holds true in any Banach space of type 2. 


\section{REFERENCES}

1. A. de Acosta and J. Kuelbs, Some results on the cluster set $C\left(\left\{S_{n} / a_{n}\right\}\right)$ and the $L I L$, Ann. Probab. 11 (1983), 102-122.

2. P. Assouad, Martingales et réarrangements dans les espaces uniformément lisses, C. R. Acad. Sci. Paris Sér. A 279 (1974), 741-744.

3. J. Diestel, Geometry of Banach spaces-Selected topics, Lecture Notes in Math., vol. 485, Springer, Berlin, 1975.

4. P. Enflo, Banach spaces which can be given an equivalent uniformly convex norm, Israel J. Math. 13 (1972), 281-288.

5. E. Giné and J. Zinn, Some limit theorems for empirical processes, Ann. Probab. 12 (1984), 929-989.

6. V. Goodman, J. Kuelbs and J. Zinn, Some results on the LIL in Banach spaces with applications to weighted empirical processes, Ann. Probab. 9 (1981), 713-752.

7. B. Heinkel, Relation entre théorème central-limite et loi du logarithme itéré dans les espaces de Banach, Z. Wahrsch. Verw. Gebiete 49 (1979), 211-220.

8. , Une extension de la loi des grands nombres de Prohorov, Z. Wahrsch. Verw. Gebiete 67 (1984), 349-362.

9. J. Hoffmann-Jørgensen, Sums of independent Banach space valued random variables, Studia Math. 52 (1974), 159-186.

10. $\ldots$, On the modulus of smoothness and the $G_{\alpha}$-conditions in $B$-spaces, Aarhus Preprint Series 1974-75, no. 2.

11. R. C. James, Some self dual properties of normed linear spaces, Ann. of Math. Studies, No. 69, Princeton Univ. Press, Princeton, N.J., 1972, pp. 159-175.

12. _ Non-reflexive spaces of type 2, Israel J. Math. 30 (1978), 1-13.

13. M. Klass and J. Kuelbs, The law of the iterated logarithm in $l^{p}$ spaces, Probability in Banach spaces. V, Lecture Notes in Math., Springer, Berlin (to appear).

14. G. Köthe, Topological vector spaces, Springer, Berlin, 1969.

15. J. Kuelbs, An inequality for the distribution of a sum of certain Banach space valued random variables, Studia Math. 52 (1974), 69-87.

16. __ A strong convergence theorem for Banach space valued random variables, Ann. Probab. 4 (1976), 744-771.

17. Kolmogorov's law of the iterated logarithm for Banach space valued random variables, Illinois J. Math. 21 (1977), 784-800.

18. M. Ledoux, Sur les théorèmes limites dans certains espaces de Banach lisses, Probability in Banach spaces. IV, Lecture Notes in Math., vol. 990, Springer, Berlin, 1983, pp. 150-169.

19. ___ La loi du logarithme itéré dans les espaces de Banach uniformément convexes, C. R. Acad. Sci. Paris Sér. I 300 (1985), 613-616.

20. M. Ledoux and M. Talagrand, Conditions d'intégrabilité pour les multiplicateurs dans le TLC banachique, Ann. Probab. (to appear).

21. J. Lindenstrauss and L. Tzafriri, Classical Banach spaces. II, Springer, Berlin, 1979.

22. B. Maurey and G. Pisier, Séries de variables aléatoires vectorielles indépendantes et propriétés géométriques des espaces de Banach, Studia Math. 58 (1976), 45-90.

23. G. Pisier, Martingales with values in uniformly convex spaces, Israel J. Math. 20 (1975), 326-350.

24. __ Un exemple concernant la super-réflexivité, Séminaire Maurey-Schwartz 1974-75, annexe 2, Ecole Polytechnique, Paris, 1975.

25. __ Le théorème de la limite centrale et la loi du logarithme itéré dans les espaces de Banach, Séminaire Maurey-Schwartz 1975-76, exposés III et IV, Ecole Polytechnique, Paris, 1976.

26. G. Pisier and J. Zinn, On the limit theorems for random variables with values in the spaces $L_{p}(2 \leq p<\infty)$, Z. Wahrsch. Verw. Gebiete 41 (1978), 289-304.

27. W. Stout, Almost sure convergence, Academic Press, New York, 1974.

28. V. V. Yurinskii, Exponential bounds for large deviations, Theory Probab. Appl. 19 (1974), 154-155.

Département de mathématique, Université de Strasbourg, 7, Rue ReneDESCARTES, 67084 STRASBOURG CEDEX, FranCE 